\title{
Encouraging Renewable Energy Consumption Through Dynamic Pricing
}

\author{
Italo C. Brito $^{*}$, Leonardo C. Ribeiro ${ }^{\dagger}$, Luiz F. R. C. Carmo ${ }^{\dagger}$, Claudio M. Farias ${ }^{\ddagger}$. Luci Pirmez ${ }^{\ddagger}$, \\ * Colégio Pedro II \\ Rio de Janeiro, Brazil 20921-903 \\ $\dagger$ National Institute of Metrology, Quality and Technology \\ Duque de Caxias, Brazil 25250-020 \\ $\ddagger$ Federal University of Rio de Janeiro \\ Rio de Janeiro, Brazil 21941-901
}

\begin{abstract}
Smart Grids are smart networks of energy transmission and distribution that enable a two-way communication between the consumer and distributor. Smart Grids are important for energy sustainability and environmental preservation avoiding the non-renewable energy consumption since decentralized suppliers are able to supply energy demands through renewable energy. It will be possible to encourage renewable energy consumption using a dynamic pricing adopting different prices to each energy source. Some network and security issues arise with that network communication like if it will be able to calculate the price in a suitable time interval. So a challenge is to analyze the impact of ICT on this operation. We analyzed the impact of a dynamic pricing on consumption changes and energy generation, and the delay time to calculate the price in a clustered network architecture.
\end{abstract}

Index Terms-Smart Grids, Real Time Pricing, Renewable Energy, Network Architecture, Security, Network Performance.

\section{INTRODUCTION}

Smart Grid is a promising technology which will modernize the actual electricity grid. One of its greatest advantages is to provide renewable energy, also called green energy, in a distributed manner. There are several renewable energy sources such as wind, small hydro, solar, rain, tides, waves, etc. Smart Grids will help to provide the continually increasing demand for energy. Also Smart Grids will make possible to avoid the non-renewable energy generation like fossil fuels, including oil, coal, and natural gas, which may harm the environment.

The Energy Information Administration [1] estimates that in 2012 the total energy consumption in the world was 19710 billion KiloWattHours (kWh) and the renewable energy consumption was 4715 billion $\mathrm{kWh}$. That means the total consumption from fossil fuel energy generation was $76.08 \%$ of the total consumption. Also the primary energy consumption has $28.01 \%$ of coal energy generation, $23.63 \%$ of gas energy generation and $34.63 \%$ of oil energy generation.

A two-way communication between the consumer and distributor is a powerful tool that can be used to reduce the use of fossil fuel energy and thus the environmental damage. Smart meters are smart devices connected to a network that meter power consumption and generation, they may send their information to a data concentrator (DC) in a short time to compute the real time consumption. The DC is a network node that stores all the information about a distribution domain. The DC can calculate the price according to the total consumption. This strategy of dynamic pricing, called Real Time Pricing (RTP), can be implemented and used to reduce the peak time consumption, and then reduce the investments on energy generation capacity [2].

Dynamic pricing models could be used to encourage the energy consumption in large renewable energy availability time [3]. A sustainability index could be used to dynamically identify the proportion of each renewable energy generation. Each smart meter that supplies energy to the grid would have a sustainability index associated, and then a DC would calculate a compound sustainability index in a distribution domain according to each energy generation. The energy footprint framework uses the sustainability index, real time consumption and generated renewable energy to calculate the price. The higher is the compound index and the total renewable energy generation the lower will be the price.

Some concerns arise from the dynamic pricing. For instance, the distributor profit may vary on each month. Another concern is if the consumer in a sporadic time is consuming more than usual, and the price gets high. The client bill may be very expensive because of additional payments. We need a strategy to limit the distributor profit variation and the client bill.

An interesting dynamic pricing model being used is RTP Day Ahead (RTP-DA) [2]. In RTP-DA, the costumer is provided with a forecast of hourly prices for each hour of the subsequent day. A Schedule RTP-DA-2 tariff in Georgia state, USA, is designed so that if the customer baseline load (CBL) accurately reflects average usage patterns, and the customer continues to follow these patterns, then the customers bill has no marginal impact from RTP prices. If the customer is able to reduce load (relative to the CBL) in periods when RTP prices are high, the customer can receive a significant bill credit.

Our goal in this paper is to analyze the impact of the dynamic pricing, specially RTP scheme, on Smart Grids to avoid fossil fuel energy generation. In this scheme, we need to grant a minimum delay to calculate the price, since the price is updated based on the consumption and energy generation at 
the exact moment. So another goal is to analyze if the network will be able to calculate the price in a suitable time interval with reliability, security and efficiency.

Each distribution scenario may use different technologies in order to perform the price calculation, and possible candidates are Power Line Communication (PLC), a data transmission based on the existing power line cables, wireless communication, cellular communication and internet based-virtual private networks [4]. We used a clustered network based on a tree topology in this work, and the technology chosen was PLC. Simulations were performed with distinct scenarios and we analyzed the peak time consumption decrease and fossil fuel energy generation decrease and later, we analyzed the delay time to calculate the price and return it to each smart meter.

The paper is organized as follows. Section II we present some related work about Demand Response, ICT architectures and security. Section III is shown the simulation details like security schemes, sequence of events to calculate the price, how to estimate consumption changes and how to calculate the delay time. In section IV, we discuss our results about the experiment. Section V describes our conclusions and future work on the experiment.

\section{RELATED WORK}

This section presents the related work to our proposal. The works presented in this section are separated in tree subsections. Demand Response \& Sustainability are works related to the costumer shifting the consumption in response to the price. Network Performance are works related to the transmission delay and network architectures. Security provide some security issues in Smart Grids (SG).

\section{A. Demand Response \& Sustainability}

First, in [5] is presented a survey of 15 experiments of household response to dynamic pricing of electricity. It's also discussed a model to estimate the costumer consumption changes: the constant elasticity of substitution (CES). The CES is an application to electricity pricing centers on the substitution of consumption.

A functionality provided by $\mathrm{SG}$ is the Energy Usage Scheduling [6]. The prices may vary in the market, so the consumer may schedule his energy usage in a lower price time. Home area networks can be deployed to connect the electrical appliances in a house to a scheduler, which activates each appliance at the appropriate time to minimize the cost of using electricity. Examples of appliances are washer, dryer, air conditioning, fan, light, and electric vehicle.

In [7] is made a study case of the benefits of demand response, the consumer profile changes in response to the prices that updates according to the energy demand. The peak time demand could potentially be reduced by $20 \%$ in Finland, for instance. It was studied the impact of demand response in 1,600 consumers in Kainnu, Finland. Results shown that just $10 \%$ of demand response potential would improve the peak system load,network loss and service reliability in 5,6\%, 1,3\% and $1,7 \%$, respectively. The potential impact of each appliance that can be scheduled the use was analyzed, and almost the entire benefits could be achieved by heating and ventilation systems.

A review of load forecasting, dynamic pricing, and demand side response (DSM) is done in [8]. DSM is an approach for planning, implementing and monitoring the energy consumption during the peak time. Some schemes of dynamic pricing discussed are Time of Use (ToU), Critical Peak Pricing (CPP) and Real Time Pricing (RTP). ToU differs the price only on peak time and off-peak time. CPP is a modification of ToU, the price is different some time of year where the energy demand is very high as compared to the rest of peak time during the year. At last, the RTP can be of two types, hourly pricing and day ahead pricing. The first can be achieved just with a good network performance, since it is a scheme the calculates the price in real time. The second one was explained above in RTP-DA-2. The load forecasting techniques consists of statistical based models or artificial intelligence that predicts the future requirements of the energy system.

An assessment of sustainability indicators for renewable energy technologies is made in [9], and instead of the hydroelectric energy gains in the indicators of price, availability and limitations, and efficiency, it loses in land use, water consumption and social impacts. So this framework could be used to avoid hydro-electric energy generation, for instance.

\section{B. Network Performance}

The current communication capabilities of the existing power systems are limited to small-scale local regions that implement basic functionalities which do not yet meet the demanding communication requirements. We need to identify the communication scenarios and characteristics in power systems and develop practically usable network solutions [6].

Some standardization were described following the lead of some organizations around the world [10]. A common standard in smart grids is the use of a Home Area Network (HAN) gateway at the users home to provide information to the grid, because other devices normally are limited in computational power. Also an architecture that uses Machine-to-Machine Communications (M2M) is discussed where SG needs a decentralized organization instead of hierarchical organization to transmit data regarding energy consumption.

A PLC communication technology used in the smart grid is the Narrow Band PLC (NB-PLC). It can be used in highvoltage transmission and reaches a distance of $150 \mathrm{~km}$ or more. Neighborhood Area Network (NAN), FAN and WAN are examples of networks that uses this technology. Advantages of PLC are that the communication infrastructure are already constructed, it has lower operation and maintenance costs, and it's possible a physical disconnection opportunity according to other networks. Disadvantages are higher signal losses and channel interference, disruptive effects caused by appliances and other electromagnetic interferences, a complex routing, and it's hard to transmit higher bit rates.

A performance analysis using PLC for AMR was made using impulsive noise interference [11]. Two different topolog- 
ical structures were used in a simulation: radial and tree. And two schemes were studied called Clustered Simple Polling (CSP) and Neighbor Relay Polling (NRP). CSP is simple because every meter sends his consumption to the DC. NRP is used when occurs a problem called "silent node", the node tries to send a packet but an impulse noise interrupts the transmission. Then, the "silent node" tries to send his packet to a neighbor, and his neighbor sends his information to the DC. Results shown that the tree topology obtained a considerable lower delay, but obtained lower throughput when both schemes are used.

The performance of NB-PLC was analyzed using a low power transmission of Internet Protocol version 6 (IPv6) to provide network reliability with acceptable latency for AMI [12]. The NB-PLC (below $500 \mathrm{kHz}$ ) supports indoor and outdoor uses and can deliver maximum of about $500 \mathrm{kbps}$. It's also analyzed the throughput (per node) using different number of nodes. Results shown that throughput of the network varies linearly with packet size. Meter reading comprising of 300 meters will require a raw throughput of $2 \mathrm{kbps}$ per node in certain cases. With a packet size of 64 bytes, the results shown a close throughput of $2 \mathrm{kbps}$ for 100,400 or 1000 meters in the network. It's concluded that the packet size is a major determinant of AMI application performance. It's also shown that services like power outage, pricing notification, event and emergency messages have the following QoS requirements for AMI: a traffic class value of high priority and critical, more than $98 \%$ packet delivery within 5 seconds and payload less than 100 bytes. The demand response applications have the following communication requirements: 14-100 kbps bandwidth per node, $99 \%$ for reliability and $500 \mathrm{~ms}$ of several latency.

In [13] is discussed Routing Protocol for Low Power and Lossy Networks (RPL) in AMI Networks. AMI networks are composed of millions of endpoints distributed across both urban and rural environments. Devices in the network communicate directly with other devices in close proximity using a variety of low-power and/or lossy link technologies that are both wireless and wired. In addition to serving as sources and destinations of packets, many network elements typically also forward packets and thus form a mesh topology. In a typical AMI deployment, groups of meters within physical proximity form routing domains, each in the order of a 1,000 to 10,000 meters. Thus, each electric meter mesh typically has several thousand wireless endpoints, with densities varying based on the area and the terrain.

A research challenge is to develop minimum-latency solutions, mainly referring to security issues. The security mechanisms provide strong protection but incur in transmission and processing delay of the message. The transmission delay should be minimum keeping the necessary security. So there is a trade-off between security performance and the total delay [6].

\section{Security}

Some security questions are discussed in [14]. A DoS or Distributed DoS attack may halt a device such as smart meters, power devices and appliances. The smart meter attacks may cause to loss of pricing data that is critical to serious economic implications. Therefore, the integrity of billing information, meter data, commands, and running software is quite important. The information privacy is provided by using cryptographic functions on the entire grid to encrypt and decrypt data. The Public Key Infrastructure (PKI) technologies are considered to meet attack detection, prevention, and privacy requirements owing to their interaction with trusted softwares. PKI includes several policies and procedures to identify customers depending to its digital certification framework that defines management, configuration, and operation strategies.

A performance comparison study of Elliptic Curve Cryptography (ECC) and Advanced Encryption Standard (AES) was made in commercial and research sensor nodes [15]. Were analyzed the Elliptic Curve Diffie-Hellmann (ECDH) and Elliptic Curve Integrated Encryption Scheme (ECIES). $\mathrm{ECDH}$ is a key agreement protocol that allows to establish a shared secret between two non-authenticated parties. On the other hand, ECIES is an encryption protocol based on EC public-key. It's also analyzed the Elliptic Curve Menezes-QuVanstone (ECMQV) scheme. This protocol is a key agreement scheme based on EC public key that supports key authentication. A Field Programmable Gate Arrays (FPGAs) was used to enhance the performance. The FPGAs serve as accelerators of complex algorithms, making it possible to process a vast amount of sensing data in real time. A chyptographic coprocessor at $12 \mathrm{MHz}$ frequency was used in the experiment. It's obtained a delay time of $0.148,0.148$ and 0.011 seconds for ECDH, ECIES and ECMQV respectively.

\section{Methodology}

This section shows how we performed our experiment in three subsections. The first one explains how we obtained the wind and solar energy generation curves and the costumer consumption curve in order to start a simulation. The second subsection describes the strategy to choose a suitable price. Finally, the last subsection gives details about the simulation like: the sequence of events, authentication process, security mechanisms and network issues.

\section{A. Consumption and Generation Curves}

We obtained data of wind speed curve from Pernambuco state of Brazil in [16]. Annual average monthly and daily average hourly wind speed were obtained from Monteiro region. The power potency curve from a wind turbine was obtained in [17]. So the daily energy generation curve was estimated using the daily average hourly wind speed combined to the power potency curve. And later, a multiplicative factor using the annual average monthly wind speed was created to increase or decrease the curve. Let $P_{M A X}$ be month with maximum capacity of wind energy generation. $P_{M}$ is the month we chose to simulate. $P_{W}$ is the maximum generation capacity 
we want. $P_{H}$ is the generation capacity on each hour. The multiplicative factor $F$ given by (1). Later, the generation capacity on each hour is calculated $P_{H}=F * P_{H}$. As a result, it's obtained the daily capacity curve of wind energy generation for each month.

$$
F=\frac{\left(\frac{P_{\mathrm{M}}}{P_{\mathrm{MAX}}}\right) * P_{\mathrm{W}}}{\sum_{i=1}^{24} P_{\mathrm{H} i}}
$$

The anisotropic diffuse solar irradiation of Botucatu city in São Paulo state was obtained in [18]. We used an efficiency of $22 \%$ of the solar irradiation for the power generation capacity. So we obtained the daily average hourly solar irradiation curve to estimate the daily solar energy generation capacity curve. And a multiplicative factor was created using the annual average monthly solar irradiation curve to obtain the daily capacity curve of solar energy generation for each month. The same equation (1) was used to obtain the solar energy generation capacity for each month.

The daily energy consumption curve and mean deviation were obtained in [19] for residential consumers with average mensal consumption between 100 and $200 \mathrm{kWh}$ of São Paulo city. In this work, we estimate that each Smart Meter (SM) will have an average monthly consumption of $150 \mathrm{kWh}$. So a multiplicative factor was created to calculate the SM average daily energy consumption curve and the mean deviation of energy consumption. Let $C_{H}$ be the energy consumption on each hour. The multiplicative factor $F_{C}$ is given by (2). Later, the energy consumption on each hour was calculated by $C_{H}$ $=F_{C} * C_{H}$. These values were used at every hour to generate random numbers from the normal distribution in order to simulate the SM consumption.

$$
F_{\mathrm{C}}=150 / \sum_{i=1}^{24} C_{\mathrm{H} i}
$$

Figure 1 shows the estimated curves for a fictitious scenario of $49 \mathrm{MWh}$ mensal consumption with a generation capacity of $50 \%$ of this demand being supplied by wind and solar energy in October. The fossil fuels energy generation is obtained calculating the difference between the total consumption and the renewable energy generation. As we can see, in this scenario there are some times the renewable energy generation capacity is higher than the consumption, specially from 9 AM to 4 PM. Assuming that there isn't enough power banks, renewable energy maximum capacity would not be used. Our goal is to reduce the peak time consumption and increase the off-peak time consumption with a great capacity of renewable energy generation.

\section{B. Price Selection}

The wind and solar energy prices were simulated varying between $0 \%$ and $100 \%$ of the original price, and the fossil fuels energy price varying between $100 \%$ and $200 \%$. The costumer behavior changes were estimated using the CES model right

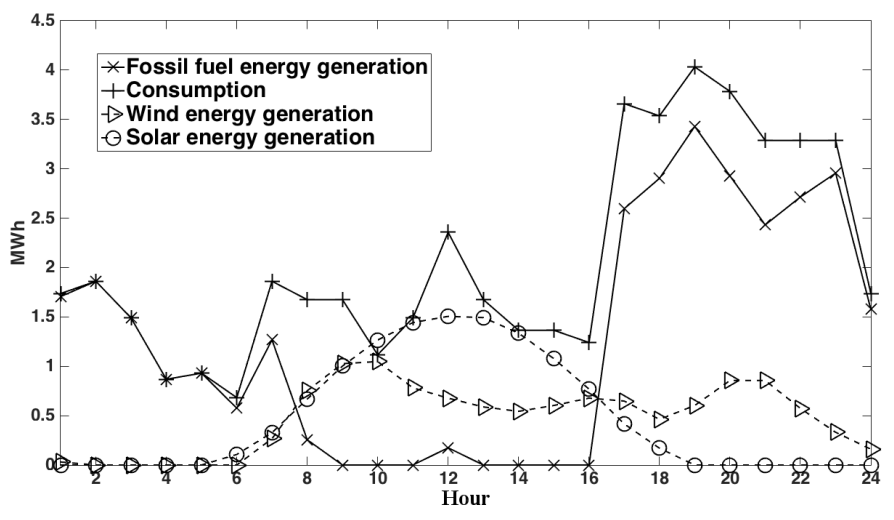

Fig. 1. Energy generation capacity and consumption curves in October

after calculated the energy price at every hour of the day. Then we compared the changed consumption to the original consumption without dynamic pricing implementation.

There is a concern to reduce the peak time consumption in order to flat the curve reducing investments on generation capacity. So it is guaranteed that after the new price this generation during the off-peak time (between $8 \mathrm{AM}$ and $4 \mathrm{PM}$ ) does not exceed the previous traditional peak time generation (between $5 \mathrm{PM}$ and $11 \mathrm{PM}$ ) on each month. It's also necessary that the distributor has a stable profit, thus the price of each kind of source energy is selected so that the variation of average profit is limited and the reduce peak time consumption is maximized.

The distributor profit may increase or decrease according to the selected prices. Thus, we selected an appropriate price for each source in order to maintain a close profit. Another concern is to limit the costumer bill. It's because in a sporadic time the costumer can consume more than usual in a high price time resulting in additional payments. A strategy could be used verifying each individual consumption curve of the costumers like in RTP-DA [2]. But this strategy will be implemented in future work.

\section{Network Simulation}

After estimated the generation and consumption curves, we begin the network simulation to calculate the price. We used a MATLAB oriented object program to create the nodes and their functionalities. In the proposed topology, each SM communicates to a Leaf Hub (LH) that concentrates the sustainability indexes and generated power. Each LH will calculate the Resultant Power (RP) and Resultant Index (RI) and will communicate to a Intermediate Hub (IH) that concentrates the same information from the LHs. Each IH communicates to a Root Hub (RH) which concentrates information about a distribution domain and calculates the price. Figure 2 shows an example of this network topology.

Because of security issues, our framework uses certificates and signatures from asymmetrical cryptography algorithms to ensure integrity, authentication, non-repudiation, and confidentiality of the packets transmitted. A PKI will help to 


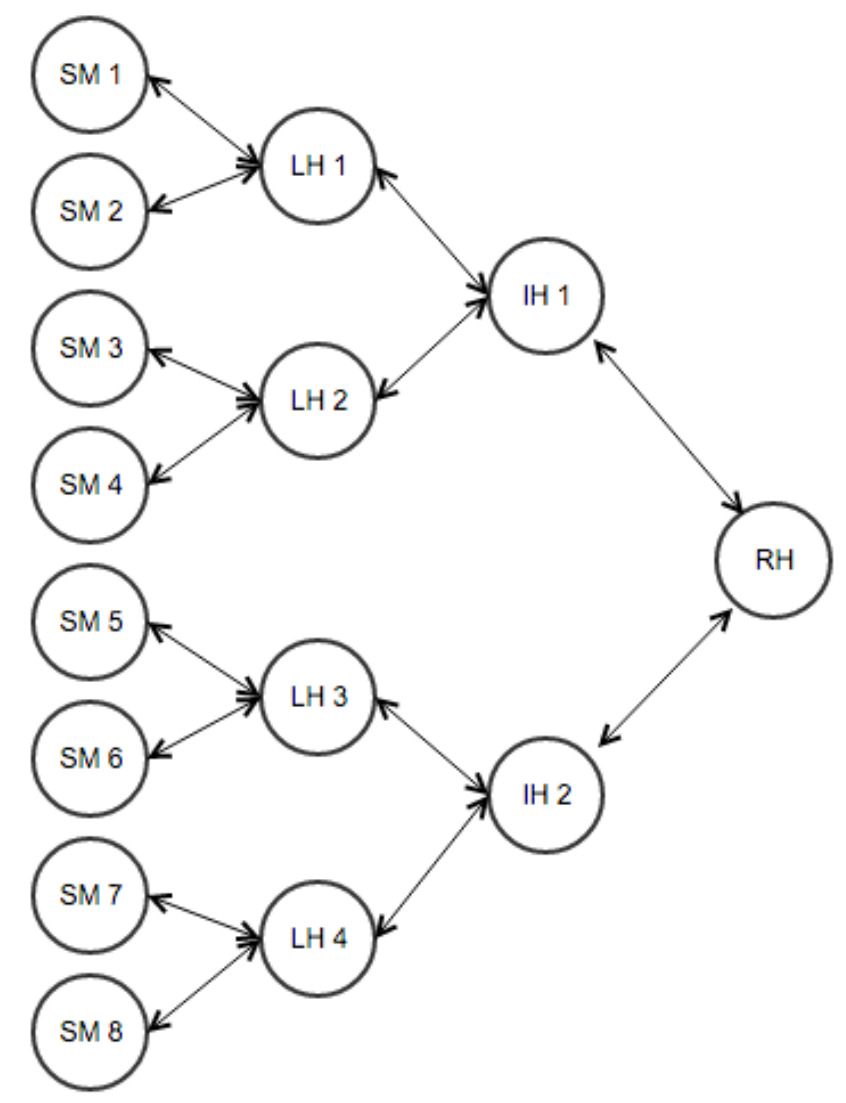

Fig. 2. Network topology communication example

authenticate the packets from a transmitter [3]. When a node is connected to the network, it needs to authenticate to the Hub that will communicate. The authentication will exchange the public key from each node. All the authentication packets will be signed by an Agency Regulatory, and the message packets will be signed by the transmitter. It's also transmitted to a DC each node sustainability index or RI in the authentication.

Some sensitive-data needs to be encrypted in the transmission like the sustainability index and the generated power by each SM in order to grant confidentiality. In this work we used the destination public key to encrypt all the sensitive-data. A timestamp is used to avoid replay attacks, in which one person with malicious intent can sniff the packet and retransmit the same packet to disturb the network.

When a receptor receives the packet, it will verify the transmitter signature with the public key to grant integrity and authenticity. The asymmetric cryptography algorithm chosen was Elliptic Curve Cryptography (ECC), which uses 20-byte keys and 20-byte signatures. Figure 3 shows the simulation events in the first time a SM will transmit packets to a LH. Figure 4 shows the simulation events in the first time a $\mathrm{LH}$ will transmit packets to a IH. The packets size are expressed in bytes. The authentication process between the IH and $\mathrm{RH}$ are the same as LH and IH. Each event in the figures represents a node executing a function. For instance, the event SM.Send is the SM node running the Send function to transmit a packet to another node.



\section{Legend}

ID : SM identification

$\mathrm{ID}_{\mathrm{H}}$ : $\mathrm{LH}$ identification

SIS: SM sustainability index

$\mathrm{e}_{\mathrm{S}}$ : SM public key

$\mathrm{e}_{\mathrm{H}}$ : LH public key

\author{
T: Timestamp \\ $\mathrm{S}_{\mathrm{RA}}$ : Regulatory Agency signature \\ $P_{s}$ : Power from SM \\ Ss: SM signature
}

Fig. 3. Simulation events between SM and LH.

Each SM sends the information packet to the LH. So the LH will calculate the RP right after receiving the last SM packet, and then will send the RP packet to an IH. The IH needs to wait every LH sending the RP packet to calculate its own RP, and then send to the RH. RH will calculate the RP and the energy price right after the last IH sending the RP.

Once the price is calculated, the RH will send the price to each IH. Right after an $\mathrm{IH}$ receives the price, it will send the price to each authenticated LH. And finally, right after a LH receives the price, it will send to each SM. All the nodes uses the same structure packet to send the price with 27.5 bytes size. Figure 5 shows the simulation events performed to return the price to a $\mathrm{SM}$.

The NB-PLC, used for long distances and high-voltage transmissions, was chosen to simulate the communications between the SG nodes. Normally it operates on the maximum frequency of $500 \mathrm{kHz}$, so we adopted a link operating at 500 $\mathrm{kHz}$ with the maximum data rate of $500 \mathrm{kbps}$ [12]. PLC has some problems to propagate through transformers, but in this work we skipped that problems.

The delay time $D_{t}$ to transmit a packet between the nodes is given by (3). We suppose that each SM has a cryptographic coprocessor of $12 \mathrm{MHz}$ using FPGAs. We adopted 0.3 seconds 


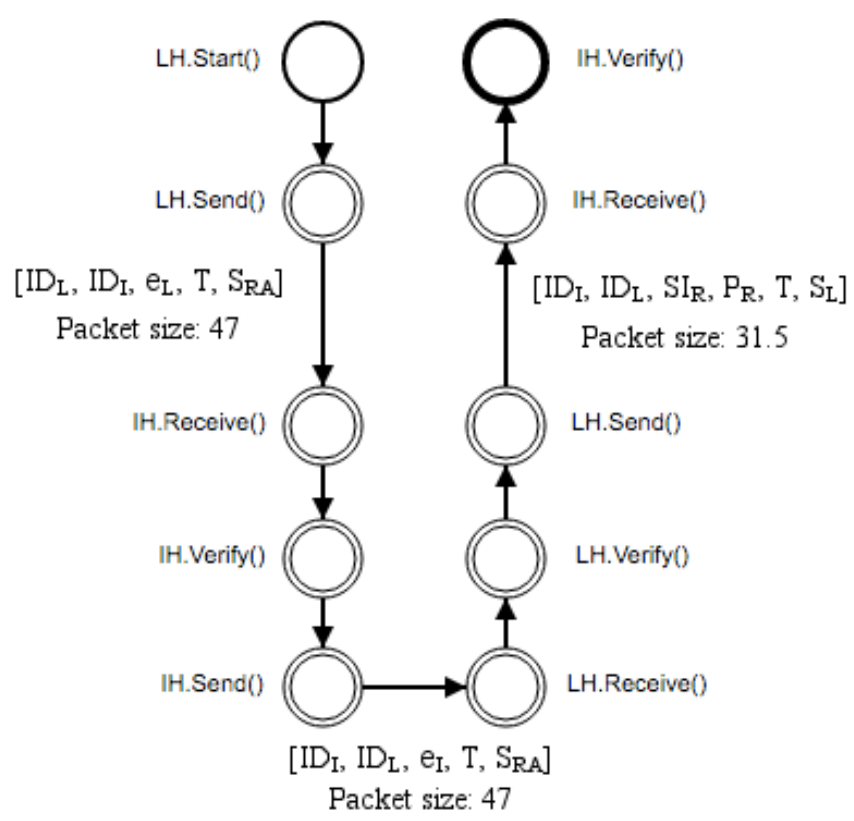

Legend

$\begin{array}{ll}\text { ID }_{\mathrm{L}}: \text { LH identification } & \text { T: Timestamp } \\ \text { ID }_{\mathrm{I}} \text { IH identification } & \mathrm{S}_{\mathrm{RA}}: \text { Regulatory Agency signature } \\ \text { SI }_{\mathrm{R}}: \text { Resultant sustainability index } & \mathrm{P}_{\mathrm{R}}: \text { Resultant Power } \\ \mathrm{e}_{\mathrm{L}}: \mathrm{LH} \text { public key } & \mathrm{S}_{\mathrm{L}}: \text { LH signature } \\ \text { e: IH public key } & \end{array}$

Fig. 4. Simulation events between Hubs.

as the processing time. Once the encryption scheme used by ECC analyzed in [15] has a delay of 0.148 seconds. Additional delay was added because of the protocol stacks of the communication technology. In future work we will include the link layer delay like header size and medium access delay like concurrence for access the medium. We will also simulate the PLC interference like impulsive and background noises.

We estimated the total time to calculate the price. Let $N$ be the number of nodes in the larger cluster. So the delay time to transmit all the power packets to a Hub is $N * D_{t}$. Then the delay time $D_{T}$ for the RH to receive the last RP packet once all the nodes are already authenticated is given by (4). $N_{S M}$ is the number of nodes in the larger SM cluster, $N_{L H}$ is the number of nodes in the larger LH cluster, and $N_{I H}$ is the number of IHs. It's also used the same equation (4) to estimate the delay for the last SM to receive the price packet.

$$
\begin{gathered}
D_{\mathrm{t}}=\left(\frac{\text { PacketSize }}{\text { LinkDataRate }}\right)+\text { ProcessingTime } \\
D_{\mathrm{T}}=N_{S M} * D_{t}+N_{L H} * D_{t}+N_{I H} * D_{t}
\end{gathered}
$$

In a real scenario of São Paulo was obtained a monthly average consumption of $49 \mathrm{MWh}$ from a distribution branch line. So we adopted $327 \mathrm{SMs}$ in the scenario to maintain a monthly average consumption of $150 \mathrm{kWh}$ per SM. The real

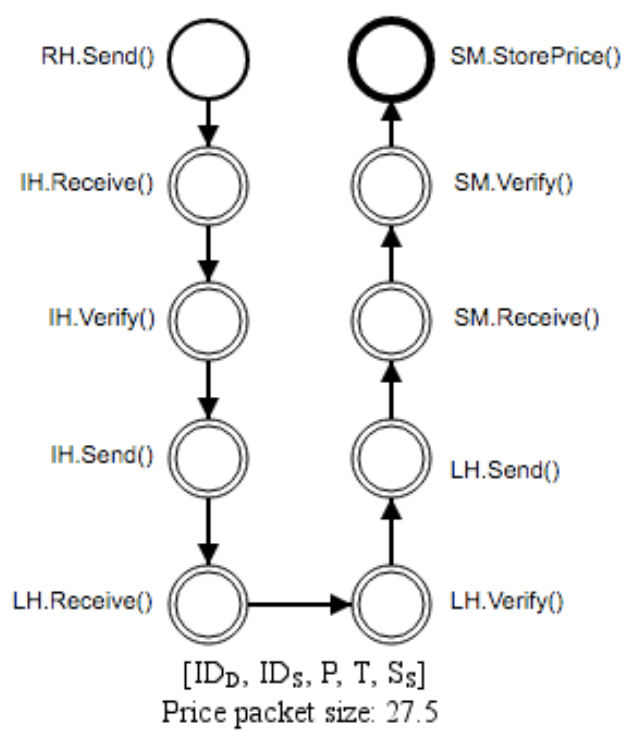

Legend

$\begin{array}{ll}\text { ID }: \text { Source identification } & \text { T: Timestamp } \\ \text { ID }: \text { Destination identification } & \text { S }_{\text {S }} \text { : Source signature } \\ \text { P: Price } & \end{array}$

Fig. 5. Simulation events to return the price to SM.

scenario has a number of 87 transformers, so we used $87 \mathrm{LHs}$ because we suppose that each $\mathrm{LH}$ will be at a transformer. And we supposed that the $\mathrm{RH}$ will be at the distribution feeder. The larger cluster size of SM and LH are 4 and 6 respectively and we varied the number of IHs in order to achieve the best performance. With these data, we could estimate that the best delay time for the last SM to receive the price packet was 13.8217 seconds and 27.6563 seconds with authentication overhead when using $10 \mathrm{IHs}$. In order to analyze the cluster size impact we simulated distinct scenarios with different numbers of SM, LH and IH.

\section{EXPERIMENTS}

This section shows our experiments results. All the experiments were made 30 times in order to achieve a 95\% confidence interval. This section is separated in two subsections. The first presents the impact of the RTP on consumption changes through selecting distinct prices to each energy source. The second shows the delay time to calculate the price in a NB-PLC transmission adopting different cluster sizes.

\section{A. Impact of Real Time Pricing}

A common objective when using Dynamic Pricing is to flat the consumption curve adopting a suitable price to reduce the investments of generation capacity. To choose a suitable price, we need to balance the renewable and non-renewable energy prices in order to maintain a close distributor profit. The renewable energy can not be so lower than the traditional because of the costumer may schedule their appliances in a time to reduce costs and we would increase the off-peak time 
consumption a lot. We don't want to shift almost the entire demand to an off-peak time.

We believe that each month or maybe each season needs to have a distinct price for each source in order to achieve the maximum reduction on peak time consumption and to encourage the renewable energy consumption on off-peak time. It is because each season or month has different renewable energy generation capacity according to wind speed, solar irradiation, ocean current, etc.

In a fictitious scenario of $49 \mathrm{MWh}$ mensal energy consumption with a generation capacity of $50 \%$ of this demand being supplied by wind and solar energy, we selected suitable prices for each month in order to shift the energy demand and maintain a stable distributor profit. We adopted two different prices: for the renewable energy and for fossil fuel energy. For instance, the renewable energy price in November could be $43 \%$ of the traditional and the fossil fuel energy price could be $132 \%$. Figure 6 shows the hourly price in November. Note that between midnight and $7 \mathrm{AM}$ the price is higher than traditional, although the consumption is low there isn't enough renewable energy available and almost the entire consumption is provided by fossil fuel energy which is more expensive. From $8 \mathrm{AM}$ to $4 \mathrm{PM}$ the price is lower because there is a lot of renewable energy available.

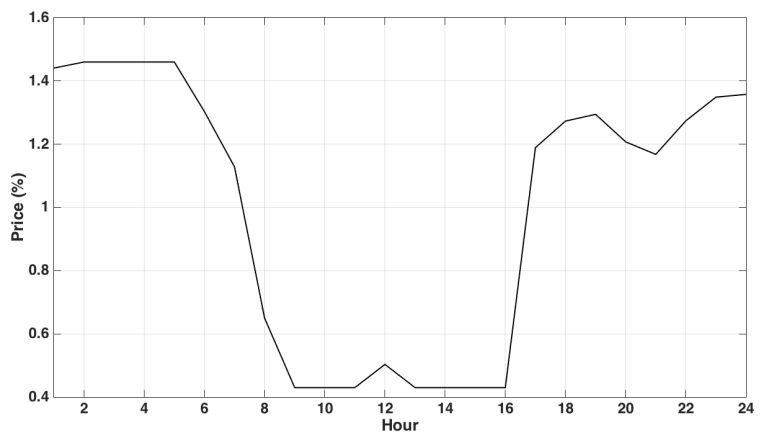

Fig. 6. Price (\%) in November.

Figure 7 shows the old energy consumption and energy generation in November. We can see that there are renewable energy lost sometimes. Figure 8 shows the new consumption and energy generation in the same period when using the selected price. Results shown that the consumption decreased 6.84 MWh on peak time and increased 9.12 MWh on off-peak time. The new consumption uses the almost the maximum renewable energy generation capacity and reduces the fossil fuel energy generation by 3.81 MWh.

Tables I, II and III shows suitable prices on each month when $40 \%, 50 \%$ and $60 \%$ of the energy consumed is from renewable sources, respectively. As we can see, the more is the domain capacity of renewable energy generation the more the prices will increase to maintain a close distributor profit.

The consumption increase and decrease is different for each month. It's because each month has a different capacity of renewable energy generation. The more renewable energy

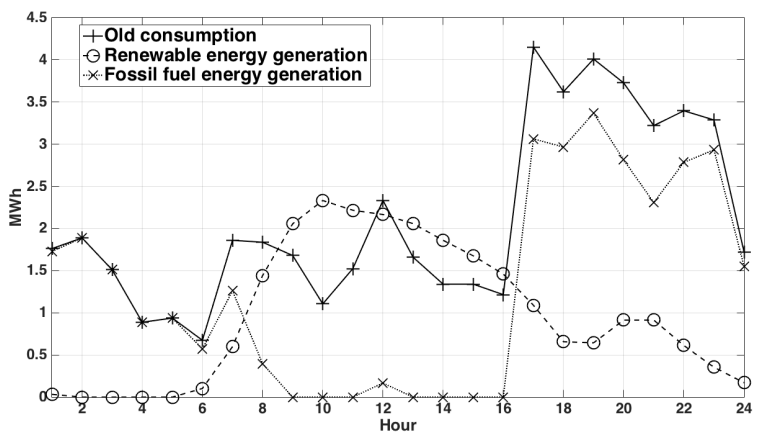

Fig. 7. Old consumption and energy generation in November.

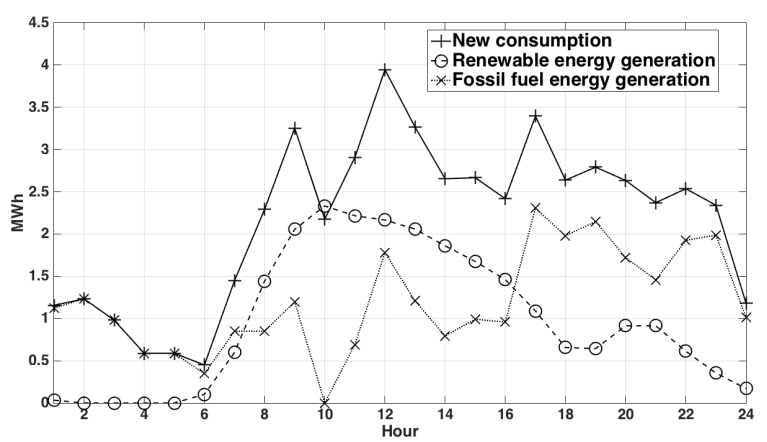

Fig. 8. New consumption and energy generation in November.

\begin{tabular}{ccc} 
Month & Fossil Fuels price $(\%)$ & Renewable price $(\%)$ \\
\hline 1 & 130 & 29 \\
2 & 126 & 24 \\
3 & 127 & 29 \\
4 & 129 & 31 \\
5 & 124 & 25 \\
6 & 122 & 25 \\
7 & 127 & 25 \\
8 & 129 & 27 \\
9 & 130 & 32 \\
10 & 137 & 35 \\
11 & 138 & 36 \\
12 & 136 & 32
\end{tabular}

SUITABLE PRICES WITH $40 \%$ OF RENEWABLE ENERGY GENERATION

\begin{tabular}{ccc} 
Month & Fossil fuels price $(\%)$ & Renewable price $(\%)$ \\
\hline 1 & 139 & 36 \\
2 & 137 & 30 \\
3 & 137 & 32 \\
4 & 137 & 29 \\
5 & 128 & 30 \\
6 & 132 & 28 \\
7 & 133 & 29 \\
8 & 139 & 34 \\
9 & 144 & 36 \\
10 & 146 & 41 \\
11 & 132 & 43 \\
12 & 121 & 32
\end{tabular}

SUITABLE PRICES WITH 50\% OF RENEWABLE ENERGY GENERATION 


\begin{tabular}{ccc} 
Month & Fossil fuels price $(\%)$ & Renewable price $(\%)$ \\
\hline 1 & 152 & 37 \\
2 & 143 & 36 \\
3 & 144 & 38 \\
4 & 143 & 44 \\
5 & 137 & 34 \\
6 & 133 & 34 \\
7 & 141 & 36 \\
8 & 144 & 35 \\
9 & 150 & 41 \\
10 & 163 & 48 \\
11 & 166 & 49 \\
12 & 152 & 48 \\
& &
\end{tabular}

SUITABLE PRICES WITH $60 \%$ OF RENEWABLE ENERGY GENERATION

generation the distribution domain has the more the price will be lower than traditional on off-peak time. And then, the consumption will decrease more on peak time and increase more on off-peak time. Figure 9 shows the consumption decrease on peak time and fossil fuel energy generation decrease using the suitable prices for a scenario which $50 \%$ of the energy is from renewable sources.

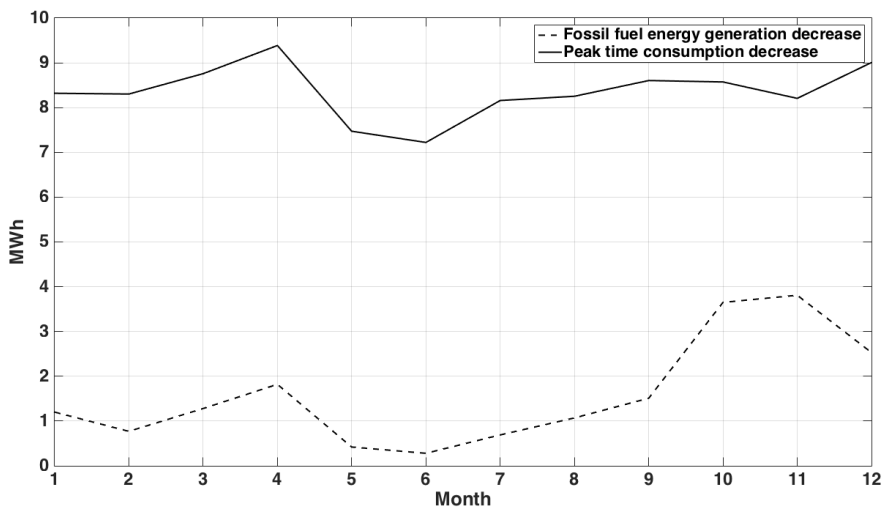

Fig. 9. Consumption decrease on peak time and fossil fuel energy generation decrease.

As the price varies on each month and the consumption changes, the distributor profit will vary. Figure 10 shows the profit along the year with the prices selected for a scenario which $50 \%$ of the energy is from renewable sources. We can see that the profit keeps stable along the year when we use the suitable prices. Note that if we use a strategy to protect the client bill, the prices may change.

\section{B. Delay Time}

To simulate a real network scenario at São Paulo we used 87 LHs and 10 IHs. We assume that the data concentrator (RH) that calculates the price will be at the substation. Each smart meter has a estimated consumption mean of $150 \mathrm{kWh}$, so we used 327 SMs. In this scenario, the delay time for the last SM to receive the price in the first hour was 27.65 seconds because of the authentication overhead. The delay time in the last hours was 13.82 seconds.

We performed simulation with different numbers of SM, LH and IH. Table IV shows the delay time to conclude a



Fig. 10. Distributor profit (\%).

calculating price cycle. For a low density distribution domain with at most 10,000 SMs the price would be calculated without many concerns. For a high density distribution domain, for instance $100,000 \mathrm{SMs}$, the number of clusters should be studied carefully.

\begin{tabular}{|c|c|c|c|c|}
\hline SM's & LH's & IH's & Delay With Authentication & Delay \\
\hline 327 & 87 & 10 & $27.65 \mathrm{~s}$ & $13.82 \mathrm{~s}$ \\
\hline 1,000 & 100 & 25 & $49.30 \mathrm{~s}$ & $24.63 \mathrm{~s}$ \\
\hline 1,000 & 100 & 10 & $38.47 \mathrm{~s}$ & $19.23 \mathrm{~s}$ \\
\hline 10,000 & 1,000 & 10 & $2 \mathrm{~m} 26 \mathrm{~s}$ & $1 \mathrm{~m} \mathrm{13s}$ \\
\hline 10,000 & 1,000 & 50 & $1 \mathrm{~m} \mathrm{38s}$ & $49.27 \mathrm{~s}$ \\
\hline 10,000 & 1,000 & 100 & $2 \mathrm{~m} \mathrm{26s}$ & $1 \mathrm{~m} 13 \mathrm{~s}$ \\
\hline 10,000 & 1,000 & 150 & $3 \mathrm{~m} \mathrm{22s}$ & $1 \mathrm{~m} \mathrm{40s}$ \\
\hline 10,000 & 1,000 & 200 & $4 \mathrm{~m} \mathrm{20s}$ & $2 \mathrm{~m} 10 \mathrm{~s}$ \\
\hline 100,000 & 10 & 5 & $3 \mathrm{~h} 20 \mathrm{~m} \mathrm{35s}$ & 1h $40 \mathrm{~m} 14 \mathrm{~s}$ \\
\hline 100,000 & 100 & 10 & $20 \mathrm{~m} 28 \mathrm{~s}$ & $10 \mathrm{~m} 14 \mathrm{~s}$ \\
\hline 100,000 & 1,000 & 100 & TABLE IV $14 \mathrm{~s}$ & $2 \mathrm{~m} 7 \mathrm{~s}$ \\
\hline
\end{tabular}

\section{CONCLUSIONS AND FUTURE WORK}

We concluded that it's possible to avoid fossil fuel energy generation shifting the demand to high availability time of renewable energy generation in order to utilize the maximum generation capacity. The price may vary seasonally and maybe monthly to achieve the maximum renewable energy consumption and to stabilize the distributor profit, but a strategy should be used to limit the client bill. In a fictitious scenario of 49 MWh mensal energy consumption that is possible to supply $50 \%$ of the energy demand by renewable sources, we could decrease the peak time consumption in 6.84 MWh and avoid the fossil fuel energy generation in $3.81 \mathrm{MWh}$ in November. In this work we avoided the fossil fuel energy generation, but in the future Smart Grid it will be possible to avoid others energy sources simply assigning a lower price.

There is a concern to achieve a minimum delay when calculating the price in a real time pricing scheme, because it needs to compute all the energy generation and consumption in a distribution domain at the exact moment. Using a NBPLC to transmit the information between network nodes, we simulated some scenarios with 327 until 100,000 smart meters. 
Also we varied the number of clusters in a distribution domain in order to get the total delay to calculate the price. Results shown that in a network distribution with high density smart meters, for instance 100,000 smart meters, it would be possible to calculate the price in 127.39 seconds using the clustered network without any interference.

Future work may simulate the NB-PLC with background and impulsive noise, and to implement the NRP scheme to collect meters data. We may include a complete protocol stack delay using other communication technologies. Another resource we may simulate is the PKI infrastructure in which a little overhead would be added to the network traffic. A wireless network could be trivial to enhance the performance and possible candidates are ZigBee and LTE. A good analysis is to implement different security schemes adopting distinct technologies to grant reliability and efficiency without performance loss.

This work is supported in part by grant from H2020-EUBR (grant no 2568).

\section{REFERENCES}

[1] U.S. Energy Information Administration "International Energy Statistics" [Online]. Available: http://www.eia.gov/cfapps/ipdbproject/IEDIndex3.cfm, accessed on Apr. 2016.

[2] Borenstein, S., Jaske, M., Rosenfeld, A. "Dynamic Pricing, Advanced Metering, and Demand Response in Electricity Markets" Center for the Study of Energy Markets, University of California Energy Institute, 2002.

[3] D. Boccardo, L. Ribeiro, R. Canaan, L. Carmo, L. Pirmez, R. Machado, C. Prado, T. Nascimento. "Energy Footprint Framework: A Pathway Towards Smart Grid Sustainability” IEEE Communications Magazine, vol. 51, no. 1, pp. 50-56, Jan. 2013.

[4] I. Colak, S. Sagiroglu, G. Fulli, M. Yesilbudak, C. Covrig. ”A survey on the critical issues in smart grid technologies" Renewable and Sustainable Energy Reviews, vol. 54, pp. 392-405, Feb 2016.

[5] A. Faruqui, S. Sergici. "Household response to dynamic pricing of electricity: a survey of 15 experiment" Springer Science+Business Media, 2010.

[6] W. Wang, Y. Xu, M. Khanna. ”A survey on the communication architectures in smart grid" Computer Networks, vol. 55, pp. 3604-3629, Oct 2011.

[7] A. Safdarian, M. Fotuhi-Firuzabad, M. Lehtonen, "Benefits of Demand Response on Operation Distribution Networks: A Case Study" IEEE Systems Journal, Vol. 10, No. 1, Mar. 2016.

[8] A. R. Khan, A. Mahmood, A. Safdar, Z. A. Khan, N. A. Khan, ”Load Forecasting, Dynamic Pricing and DSM in Smart Grid: A Review" Renewable and Sustainable Energy Reviews, Vol. 54, Feb. 2016.

[9] A. Evans, V. Strezov, T. J. Evans, "Assessment of sustainability indicators for renewable energy technologies" Renewable and Sustainable Energy Reviews, Vol. 13, Jun. 2009.

[10] M. Albano, L. L. Ferreira, L. M. Pinho. "Convergence of Smart Grid ICT Architectures for the Last Mile" IEEE Transactions on Industrial Informatics, vol. 11, no. 1, pp. 187-197, Feb 2015.

[11] B. Sivaneasan, E. Gunawan, P. L. So. "Modeling and Performance Analysis of Automatic Meter-Reading Systems Using PLC Under Impulsive Noise Interference" IEEE Transactions on Power Delivery, vol. 25, No. 3, pp. 1465-1475, July 2010.

[12] A. Ikpehai, B. Adebisi. "6LoPLC for Smart Grid Applications" IEEE International Symposium on Power Line Communications and Its Applications (ISPLC), Apr 2015.

[13] D. Popa, M. Gillmore, L. Toutain, J. Hui, R. Ruben, and K. Monden "Applicability Statement for the Routing Protocol for Low Power and Lossy Network (RPL) in AMI" Internet-Draft of the Internet Engineering Task Force (IETF), version 12, March 2016.

[14] Y. Kabalsi. "A survey on smart metering and smart grid communication" Renewable and Sustainable Energy Reviews, vol. 57, pp. 302-318, May 2016.
[15] A. Piedra, A. Braeken, A. Touhafi. "A Performance Comparison Study of ECC and AES in Commercial and Research Sensor Nodes" IEEE EuroCon, July 2013.

[16] F. J. L. Lima, E. P. Cavalcanti, E. P. Souza, in Portuguese. "Avaliação do Potêncial Eólico em Cinco Regiões do Estado da Paraíba” Revista de Geografia, 2010.

[17] A. P. Petry, J. M. L. Mattuella, in Portuguese. "Análise do Potencial Eólico e Estimativa da Geração de Enegia Empregando o Software Livre Alwin” Technical Report, 2007.

[18] A. D. Pai, J. F. Escobedo, in Portuguese. "Série Temporal Diária Média Horária e Anual Média Mensal Diária da Irradiação Solar Difusa Anisotr pica” Energia na Agricultura, 2006.

[19] A. A. Francisquini, in Portuguese. "Estimação de Curvas de Carga em Pontos de Consumo e em Transformadores de Distribuição” Masters dissertation, UNESP, Ilha Solteira, 2006. 